\title{
Evidence-Based Medicine. A Lesson for Electrocardiography?
}

\author{
Ljuba Bacharova
}

Bratislava, Slovak Republic

Electrocardiography is an old diagnostic method, introduced at the end of the 19th century. Now, at the beginning of the 21 st century, one of the main concerns of electrocardiologists is a decreased interest in electrocardiography in general, particularly among young scientists ${ }^{1}$.

Naturally, electrocardiography has strengths and weaknesses related to its age. On the one hand, it is a wellestablished, accepted, and respected clinical and experimental method, based on a substantial body of knowledge and experience, accumulated during the century of its existence. On the other hand, limitations also exist related to this age: fixed out-of-date ideas and beliefs, modifications caused by rewriting and recitations, and a gap between fixed ideas about ECG and up-to-date nonelectrocardiologic knowledge.

In terms of marketing, the interest in electrocardiography displays the typical curve of a product life cycle (fig. 1): a gradual introduction of clinical diagnostics at the beginning of the 20th century, when the first experience was collected, analyzed, and related to physiological and pathological processes; a steep growth in interest, when the added value of ECG to clinical decision making was recognized and applied in many situations. Electrocardiography at that time was one of few objective methods available in cardiology and contributed substantially to the improvement in diagnostics, ie, of myocardial infarctions and hypertrophies, and introduced new diagnostic categories, such as pre-excitation or conduction defects, and a detailed classification of arrhythmias; the maturity of demand, when ECG was already clinically well established, and other diagnostic methods were not yet introduced; now, we are facing a decline in interest, with electrocardiography gradually being re-

International Laser Center.

International Laser Center, Ilkovicova 3, 81219 Bratislava, Slovak Republic. Mailing address: Ljuba Bacharova - International Laser Center - Ilkovicova 3 81219 Bratislava - Slovak Republic - E-mail: bacharova@ilc.sk

Received to publication on $15 / 4 / 02$

Accepted in 15/1/03 placed by new imaging methods in many applications. And in some applications, ECG is even considered obsolete, eg, in the diagnostics of left ventricular hypertrophy $(\mathrm{LVH})$.

The rational way to increase the interest of cardiologists, to attract young scientists, and to increase the value of ECG, is to analyze and consequently to reduce its limitations. This means, first, to reevaluate the "old truths" and "myths," second, to focus attention on the unique information provided by electrocardiography, and third, to recognize the added value of ECG in the context of other diagnostic methods, such as echocardiography, scintigraphy, NMR, and many others. I do not consider the frequently presented statement that ECG is easily available and inexpensive to be a sound argument.

The need to constantly reevaluate accumulated knowledge and evidence is not limited to electrocardiography. It is a necessity for most fields of medical and professional practice. In the last 2 decades, this need has been formulated as evidence-based medicine (EBM) demanding the critical appraisal of evidence. Sackett et $\mathrm{al}^{2}$ defined evidencebased medicine as the conscientious, explicit, and judicious use of current best evidence in making decisions about the care of individual patients. The practice of EBM means integrating individual clinical expertise with the best available external clinical evidence from systematic research. The evidence-based approach includes 4 stages: the formulation of answerable questions; the search for the best evidence; critical appraisal of the evidence; the decision to apply the conclusions to patients' health care.

EBM provides general principles and a standardized way to reevaluate knowledge. The aim of this article is to apply the principles of evidence-based medicine to electrocardiography and to begin discussions about evidencebased electrocardiography. An evidence-based medicine approach is applied to the ECG diagnostics of left ventricular hypertrophy based on voltage criteria not currently considered a "hot topic" and one in which a decline in interest is evident. We will assess whether an EBM approach is helpful in our efforts to reevaluate the "old truths," to define the unique information provided by electrocardiography and 


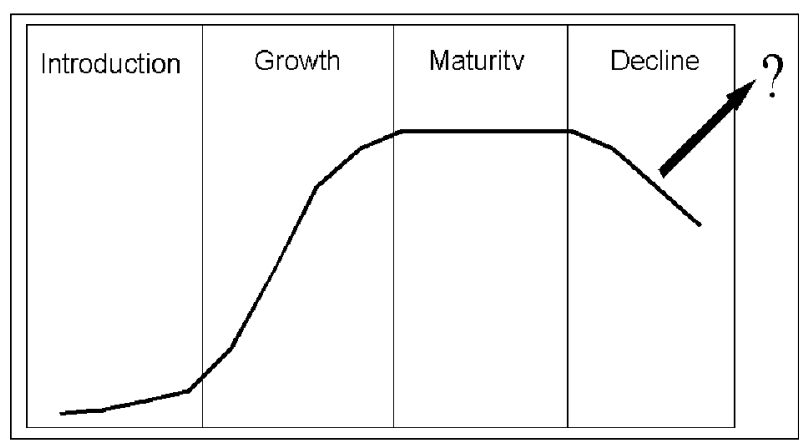

Fig. 1 - The product life cycle: a message for electrocardiography. The introduction of electrocardiography to clinical diagnostics - the collection of the first experience, analysis, and interpretation of electrocardiograms in relation to physiological and pathological processes. The growth of the interest - electrocardiography, at that time one of a few objective methods available in cardiology, contributed substantially to the improvement in diagnostics, introduced a detailed classification of arrhythmias and new diagnostic categories, such as pre-excitation and conduction defects. The maturity of demand - electrocardiography is clinically well established, and other diagnostic methods are not yet introduced, or their clinical utilization is at its beginning. The decline of interest - electrocardiography is gradually replaced by new imaging methods in many applications. How to overcome the decline? What is the added value of electrocardiography for clinical diagnostics, prognosis, and research?

the role and its added value per se of ECG, and its value in the context of other diagnostic methods.

\section{Left ventricular hypertrophy and the ECG diagnostics of $\mathbf{L V H}$}

The basic general definition of hypertrophy is an " $i n$ crease in the size of an organ or tissue without an increase in the number of its component units. Hypertrophy of the heart may result in a several-fold increase of in size and functional activity without a change in the number of muscle fibres. Often the term is loosely used to mean an increase in size without regard to the unit of structure" " We can define LVH as an increase in LV size and functional activity. This increase in size of individual myocytes and of the left ventricle as a whole is conditioned and associated with structural and functional changes, such as gene expression, protein synthesis, intracellular morphology, gap junction distribution, energy supply, oxygen consumption, structural rearrangement of myocardial tissue, contractility, apoptosis, etc. It needs to be stressed that LVH is not a static phenomenon, but it develops and progresses over time, in extreme cases, leading to heart failure. Three distinct stages of experimental LVH with characteristic biochemical, subcellular, cellular, tissue, and functional changes have been described $^{4,5}$ : The first stage represents the period of adaptation and the rebuilding of myocardium to achieve adequate performance, the second stage is defined as compensated hypertrophy, and during the third stage a gradual development of heart failure and degradation of the myocardium occur. The complex rebuilding of structure is sometimes referred to as anatomical remodeling and in relation to electrogenesis as electrophysiological remodeling.

Therefore, in the case of LVH, we have to consider: increase in size, anatomical and electrophysiological remodeling; progress and changes over time.
Evidence-based medicine. A lesson for electrocardiography

However, probably due to limited clinical diagnostic possibilities, the definition of LVH is restricted to the increase in size or mass. For example: "The most valid definition of hypertrophy is 'a demonstrable increase in isolated $L V$ muscle mass" 6 .

The electrocardiographic diagnosis of LVH is primarily based on so-called voltage criteria: the increased amplitude of the QRS complex in selected leads. The increased voltage of the QRS complex is considered a specific diagnostic finding for $\mathrm{LVH}^{7-10}$.

The current underlying hypothesis expresses the persistent belief that an increased myocardial mass should produce a stronger cardiac electric field and should be reflected in the increased voltage of the QRS complex. For example: "The excitation of the larger and thicker muscle mass results in larger and longer living activation boundaries, which in turn, result in the more than usual preponderance of the leftward and posteriorly oriented electrical forces" "11. Or: "The greater LV mass causes an increase in QRS amplitude..." The electrocardiographic diagnosis of LVH is based mainly on the increase in QRS voltage generated by the increase in LV mass" ${ }^{12}$.

The generally accepted formula for the magnitude of the extracellularly recorded potential $E$ is based on the solid-angle theory ${ }^{13}$ :

$$
E={ }_{4 \pi}^{\Omega} \Delta V m^{*} K
$$

where $\Omega$ is the solid angle, relating the boundary geometry to the electrode site. $\Delta V m$ is the transmembrane potential difference, $K$ is a constant related to the conductivity and anisotropy of the myocardium. Theoretically, the potential at the recording site is determined by the conductivity of the medium, the charge density per unit area of the polarized surface, and the solid angle subtended at the recording site by this surface ${ }^{14}$.

However, using voltage criteria, electrocardiography detects only a small percentage of the cases of LVH documented by the echocardiogram or by autopsy ${ }^{10,15}$. The low performance of voltage criteria is a source of strong criticism of ECG in LVH diagnostics and has been leading gradually to decreased interest in electrocardiography among cardiologists.

\section{The lesson to be learned from evidence-based medicine}

1) The formulation of answerable questions - Currently, the continuous effort of electrocardiologists is directed towards the best estimate of left ventricular size or left ventricular mass (LVM), to the detection of increased LVM or size. In other words, it means that we ask the following questions: "How big is the left ventricle?" or "Is the left ventricle bigger than a healthy one?"

However, electrocardiography does not primarily provide the information on the size or mass. The information provided by electrocardiography is on the electric field generated by the heart and recorded on the body surface. 
The magnitude of recorded potentials can, but does not necessarily, reflect the size of the source (fig. 2). In other words, sources of different size can exhibit the same voltage, if they differ in their electrical properties. It follows, that we are asking a question, which is not answerable.

The questions, which could be answerable by electrocardiography, are: "How strong is the LV as a source of cardiac electric field?" Or more focused: "How strong is the LV as a source of cardiac electric field in relation to its size?"

2) The search for the best evidence - The range of publications on ECG diagnostics of LVH is extensive. The increased QRS voltage was reported as early as 1906 by Einthoven ${ }^{16}$, as characteristic of LVH. From this time, a variety of electrocardiographic criteria for detection of $\mathrm{LVH}$ have emerged over the years and a number of recommended criteria have been published. The development of ECG voltage criteria has followed the development of ECG methods, from the standard 12-lead ECG where frontal plane and horizontal plane individual leads, combined criteria, or the total sum of the QRS have been used, through orthogonal electrocardiography and vectorcardiography, to the evaluation of body surface potential mapping.

A natural tendency is to read more recent publications that provide the latest evidence and opinion. Many historical publications are not readily available. However, the recency of publications could lead to unexpected surprises, as can be demonstrated by quotations about the Brody effect.

The original text ${ }^{17}$ : The relatively large conductivity of the intracavitary blood mass tends to short-circuit the tangential components, but augments the radial components of myocardial doublets, reflected in the decrease of tangentially oriented dipole moment and in the increase of radially oriented dipole moment.

A slight shift in terms - from dipole moment to electrocardiographic surface potential:

"Brody, using a mathematical model, suggested that intracardiac blood, a highly conductive mass, augments the electrocardiographic surface potential if the progress of myocardial excitation is radial to the blood mass. ${ }^{18}$

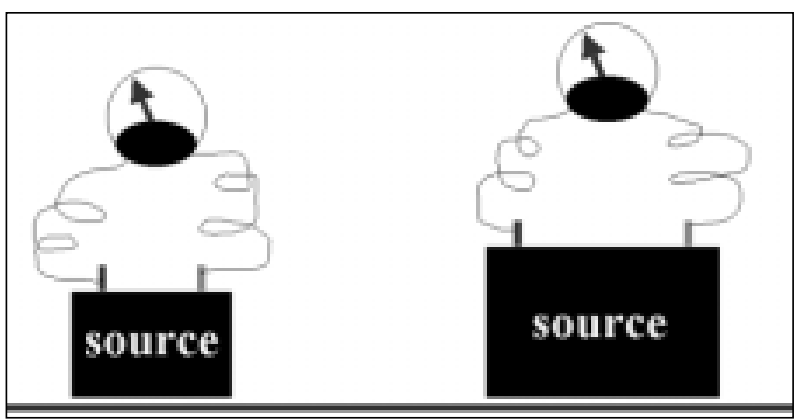

Fig. 2 - The information provided by electrocardiography is on the electric field generated by the heart. Equal voltages can be recorded at the body surface, generated by sources of different sizes, if they differ in electrical properties. Therefore, the answerable questions can be for example: "How strong is the left ventricle as a source of the cardiac electric field?" or more focused: "How strong is the source of the cardiac electric field in relation to its size?"
A shift in meaning - a conductivity-volume shift: intracavitary conductivity resulting from increased blood volume.

"Bayley and Berry (1963) have suggested that increased intracavitary conductivity resulting from increased blood volume will augment the QRS voltage by local effects on the dipoles in the cardiac muscle" ${ }^{19}$.

One interpretation of the Brody effect: An increase in QRS amplitude with decreasing volume:

"This finding would support the Brody effect, namely, an increase in QRS amplitude with a fall in end diastolic volume" ${ }^{20}$.

And an opposite interpretation: An increase in QRS amplitude with increasing volume and a reduction in QRS amplitude with a decrease in volume:

"Therefore, according to the Brody effect, an increase in left ventricular volume should be accompanied by an increase in surface QRS amplitude, and a decrease in volume, by a reduction in amplitude" ${ }^{21}$; or "According to Bonoris, $R$ wave increase during exercise should be ascribed to an increase in chamber size in patients with coronary artery disease, whereas no change occurs in normal subjects: this is so called [the] 'Brody effect" " ${ }_{22}$; or "Brody has reasoned that a dilated ventricle will more effectively augment radially directed dipoles because of the increase in intracavitary blood mass and thereby increase the voltage inscribed at the body surface" ${ }^{23}$.

Using suitable selected quotations, one can explain both an increase and a decrease in QRS amplitude due to both changes in conductivity or in increased or reduced volume, respectively, simply by the application of the Brody effect.

In spite of the variety of voltage criteria, one theme is common - the search for the increased QRS amplitude, exceeding the upper normal limits. Only values exceeding the upper normal limits are considered evidence. It is reflected also in the terminology used; these results are called "true-positive". The values within normal limits - lower than expected - are earmarked as "false-negative" and are neglected in clinical diagnostic or prognostic decision making. However, these so-called false- negative findings are the dominant findings in the ECG diagnostics of LVH. In other words, enough evidence exists that the voltage in LVH is in the majority of cases lower than that expected according to the classical hypothesis.

3) Critical appraisal of the evidence - In this part, the focus will be on the critical appraisal of the current classical hypothesis, the application of the solid angle theory, the design of studies and of argumentation and derived conclusions in these studies.

\section{Critical appraisal of the tested hypothesis}

As already mentioned above, according to the classical hypothesis we expect that the increased mass of the left ventricle in LVH generates a stronger cardioelectric field. This assumption is rather straightforward: the bigger the mass, the more severe the hypertrophy, the higher the amplitude of QRS should be. 
This hypothesis was derived from the following clinical and experimental evidence: the increased QRS amplitude exceeding upper normal limits is seen in a proportion of patients with LVH and is highly specific for LVH; close correlations between QRS amplitude and hemodynamic data of patients with left ventricular overload have been reported.

Moreover, the increased voltage of the QRS complex was revealed as an independent significant risk factor for cardiovascular mortality ${ }^{24}$. These findings stress the prognostic value of increased voltage and provide additional arguments in favor of the importance of increased QRS amplitude.

However, enough evidence exists biasing the validity of this hypothesis. Of particular relevance here is the high proportion of false-negatives, resulting in a low sensitivity of voltage criteria in $L V H$ detection ${ }^{25}$. Reported sensitivities are mostly below $50 \%$, ranging to $20 \%$, or even lower ${ }^{26-28}$. It means, on the other hand, that the percentage of false-negative results is over $50 \%$, up to $80 \%$ and even more. Next, the modest correlation between $\mathrm{QRS}$ voltage and left ventricular mass results in an unsatisfactory estimation of LVM. In spite of the fact that the proportion of patients with increased QRS amplitude is relatively small, these findings are generalized and it is expected that all patients should have increased voltage. If not, it is concluded, that ECG is of limited value or even obsolete.

Mashima ${ }^{29}$ called hypertrophy causing the enlargement of the QRS amplitude as "ideal" hypertrophy. Ideal hypertrophy refers to a condition where the ventricular muscle increases its volume without any alteration in the activation sequence, and the strength of the double layer and the velocity of the activation wave are the same as normal. In other words, the electrical properties of hypertrophied myocardium are the same as those of healthy tissue.

It follows that the current hypothesis has 2 implications and therefore is valid only if the following 2 conditions are true: no difference exists between the electrical properties of hypertrophied and healthy myocardium; the electrical properties of hypertrophied myocardium do not change during the evolution and progress of LVH.

Experimental and clinical cardiology provide enough evidence that these 2 conditions are not true. First, the pathologically changed myocardium in LVH differs from the healthy one at molecular, subcellular, cellular, and tissue levels. Structurally altered myocardium inevitably changes the conductivity properties of the tissue. Roberts et al ${ }^{30}$ showed that the wave of activation is not uniform in its rate of spread or its density. Recently, these changes have been extensively studied especially in relation to arrhythmogenesis. Disease- (and arrhythmia-) induced changes in cardiac electrical properties are referred to as electrophysiological remodeling ${ }^{31,32}$. So, an interesting dissociation in clinical thinking can be observed. In relation to arrhythmogenesis, both interrelated anatomical and electrogenic changes in $\mathrm{LVH}$ are taken into account, and the changes in electrogenesis are considered a substrate for re-entry circuits, whereas in the case of voltage criteria, we still think only in terms of anatomical changes.
Evidence-based medicine. A lesson for electrocardiography

Second, anatomical and related electrophysiological remodeling depends on the evolution and progress of LVH. As mentioned before, 3 distinct stages of experimental LVH have been described, with characteristic biochemical, subcellular, cellular, tissue, and functional changes ${ }^{4,5}$ : The first stage represents the period of adaptation and the rebuilding of myocardium to achieve adequate performance, the second stage is defined as compensated hypertrophy, and during the third stage a gradual development of heart failure and degradation of myocardium occur. However, we are estimating only the linear correlation between left ventricular mass and QRS amplitude.

In summary, evidence indicates that the hypertrophied myocardium is pathologically changed and that these changes develop/change over the time.

Mashima ${ }^{29}$ pointed out that discrepancies in actual cases indicate deviations from the ideal state. Till now, discrepancies have been attributed mainly to extracardiac factors that influence physiological variability, such as age, gender, race, anthropometric variables, intracavitary blood volume, conductivity properties of intrathoracic organs, location of the heart within the thorax, and other such things ${ }^{33}$. However, even the most sophisticated ECG criteria, which combine all known factors influencing interindividual variability, have nearly the same discriminative power ${ }^{15}$. Or, the deviations are attributed to the coincidence of pronounced cardiac pathology: ischemia, edema, and heart failure. But, the false-negative results are also seen in initial and mild hypertrophy ${ }^{34,35}$.

In summary, the gaps in our knowledge are related mostly to these deviations: what is the pathophysiological background for the deviations from "ideal" hypertrophy, ie, between the increased mass and QRS voltage lower than expected? How do these deviations change over time with the progress of LVH? How do they change with therapy? What is their diagnostic and prognostic meaning?

\section{Critical appraisal of the application of the solid angle theory}

The solid angle theory defines in biophysical terms the magnitude of a potential detected at an extracellular site. Part of the formula $\frac{\Omega}{4 \pi}$ represents the spatial determinant of the recorded voltage. It is determined by the actual geometry of the boundary and the electrode distance from the boundary. The rest of the formula represents nonspatial determinants and characterizes the electrical properties of myocardium. The nonspatial determinants include the transmembrane voltage difference, the difference in conductivity and in homogeneities within cardiac muscle. However, in the application of the solid angle theory in cases of LVH, only spatial determinants are considered. The interplay of nonspatial determinants is not considered at all. So, similarly to the hypothesis, in the application of the solid angle theory to LVH, the focus is on the size of the left ventricle. 
And consequently, the gaps in knowledge arising in relation to the appraisal of the application of the solid angle theory are related to the nonspatial determinants: are the individual nonspatial determinants unchanged during the progress of LVH? Are they changed, but balanced with others, so that they look unchanged as a whole or at the macro level? Are they unchanged with therapy?

\section{Critical appraisal of the design of the studies}

In the design of the studies, several sources of potential biases can be identified related to: the selection of the gold standard; the criteria used for inclusion of the patients into control/normal and LVH groups, respectively; the absence of the control of patients for therapy; the absence of the control of patients for known extracardiac factors influencing the QRS amplitude; time difference between recorded ECG and the gold standard method of measurement.

The gold standard method should allow distinction between subjects with or without a certain pathology, in our case subjects with or without left ventricular hypertrophy. Currently, echocardiography is considered the optimal gold standard ("the nonelectrocardiographic independent method"), mainly for its good estimation of LV dimensions and mass, documented by a close correlation with autopsy findings ${ }^{36,37}$. The fundamental gold standard is considered the autopsy, where dimensions and mass can be directly measured. The primary information of both methods is the information on size and mass, respectively, and so LVH diagnostics are often simplified at the detection of the increased mass/size. However, using only size as a measure of LVH could be misleading and a source of potential biases. Firstly, the increased mass/size is really a dominant characteristic, but only ONE of the characteristics of LVH, not the ONLY characteristic. Secondly, taking into account only the mass/size, pathological reasons for increased mass/size other than LVH and associated diseases can be overlooked. And on the other hand, the initial stages of LVH, when the mass does not exceed the arbitrary upper normal limit, can go unrecognized and therefore may be misclassified. And finally, ECG does not provide information on size or mass. It provides information on one of the functional characteristics of the heart - electrogenesis. Therefore, it would be more appropriate to use terms like normal function or adequate function in relation to the size, hypo- or hyperfunction in relation to the size of LV (fig. 3). The endless calculations of specificity and sensitivity, or derived receiver operating characteristics curves, can only repeatedly confirm the well-known fact that the size of an organ and its function are not necessarily proportional.

The simplifications in considering LVH as only an increase in mass/size are reflected in some studies in the criteria used for inclusion of the patients into control/normal and LVH groups. For example, a variety of cardiovascular pathologies included both control and LVH groups ${ }^{38}$. An unknown proportion of patients with mild hypertension is included in the normal control group, and the LVH group contains an unknown proportion of "normotensive patients" ${ }^{39}$ are they healthy subjects or hypertensive patients on therapy?

In most of the studies, the groups are not controlled for therapy. This study includes an unstated assumption that therapy has no influence on the recorded QRS voltage.

Similarly, the groups are not controlled for known extracardiac factors influencing the physiological variability of QRS amplitude, such as age, sex, anthropometric characteristics, or race. Extracardiac factors were documented to influence the QRS amplitude ${ }^{40-43}$. But curiously, in the interpretation they are mostly used to explain the lower amplitude, the false-negative results in LVH patients. However, they should influence both normal and LVH population in the same directions, if the studies are properly randomized.

The time difference between recorded ECG and the gold standard method of measurement can be considerable, especially in studies using the autopsy as a reference method. The reported intervals from the interpreted electrocardiogram until death go up to 11 weeks ${ }^{44}$, or are not precisely defined ${ }^{15,45}$. This study design means that no changes in QRS amplitude are assumed over the time interval between the recordings of the 2 methods.

\section{Sources of flaws in argumentation and derived conclusions}

The argumentation used in the ECG diagnostics of $\mathrm{LVH}$ is rather monotonous. It assumes that the "electrical dominance" of the left ventricle is enhanced in LVH. So it searches for the evidence-for increased QRS amplitude and only these results are considered TRUE. This effort is

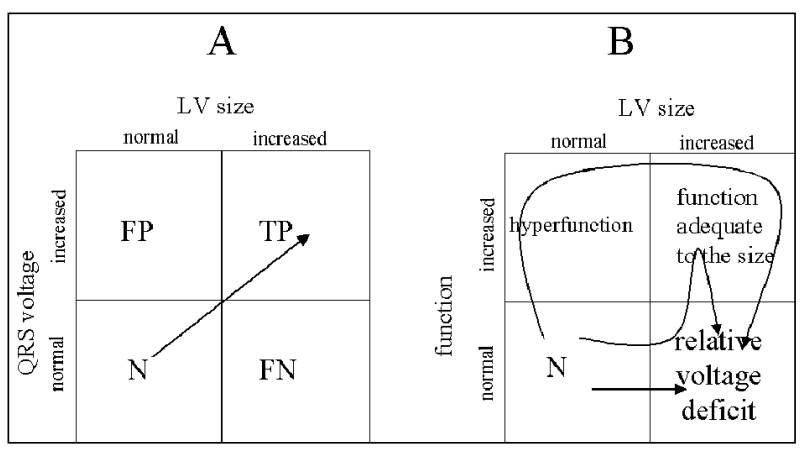

Fig. 3 - A: The classical contingency table relates the size of the left ventricle and QRS amplitude. The area of the table is divided by the upper normal limits of both methods into 4 areas: N: the area of normal results; TP: true-positive results, where an agreement between size and voltage is achieved; FP: false-positive results; FN: falsenegative results. The arrow expresses that a linear relationship between the size and the voltage is expected, in other words, the bigger the left ventricle, the higher the QRS voltage. This design of the contingency table implicitly means that the tested method and the gold standard method are alternative or competing methods. B: Our approach equally considers both function and size. The dominant findings in left ventricular hypertrophy, so-called false-negative results, those with increased mass and QRS voltage within normal limits, are according to this approach defined as the "relative voltage deficit". In this arrangement, echocardiography and electrocardiography are neither alternative nor competing methods, but complementary methods, bearing different and complementary information on diagnosis, prognosis, and the effect of therapy. The relationships between size and function during the progression of LVH are not linear, but may follow different trajectories. 
theoretically supported by the partial application of the solid angle theory, where only spatial determinants are mentioned. Findings with QRS amplitude within normal limits are false-negative. If the proportion of these false-negative results is "acceptable" or the tested criteria "significantly improve" their sensitivity, which could be really of low clinical relevance, the tested ECG criteria are recommended as suitable for ECG diagnostics of LVH. If the proportion of the false-negative results is not acceptable, and the proportion of the false-negative results was documented as high, up to $90 \%$, it is concluded that ECG is of limited value for $\mathrm{LVH}$ diagnostics or even an obsolete method.

A few weak points can be identified in this sequence of reasoning. One of the possible flaws, the generalization, has already been mentioned. If the increased QRS amplitude is found in a proportion of patients, it does not necessarily mean that it is true for all patients. The majority of ECG findings in LVH are within normal limits, which implies regularity.

The application of the spatial angle theory is not complete, only partial, because only spatial determinants are mentioned. Nonspatial determinants are not considered, even when the changes in conductivity are explicitly discussed in relation to the prolongation of QRS duration in the same article, which can be seen in reports on the voltageduration product ${ }^{38}$. The negligence of nonspatial factors is based on the findings of studies where no changes in the transmembrane action potentials in myocytes of chronically hypertrophied hearts were reported ${ }^{46-48}$. Consequently, it has been concluded that the geometry of the ventricle plays an important role in determining the increased voltage ${ }^{23}$. However, enough experimental and clinical evidence are available indicating that nonspatial determinants can change during the progression of $\mathrm{LVH}$. The electrical properties of myocardium are extensively studied in relation to arrhythmogenesis. A great amount of accumulated knowledge about the changes in active and passive electrical properties of myocardium in $\mathrm{LVH}$, such as heterogenous changes in action potential duration and amplitude, loss or thickening of gap junctions, expression and distribution of connexin ${ }^{43}$, intercellular coupling, changes in conduction velocity, interactions between ionic currents and cardiac microstructure ${ }^{49-57}$, which are sometimes referred to as electrophysiological remodeling.

Experimental and clinical evidence exists that the unstated assumptions mentioned previously are not necessarily true. The electrical properties of myocardium, which can influence QRS amplitude, can change during the progression of $\mathrm{LVH}$, with therapy and over time and can counterbalance the influence of the increased spatial angle.

And finally, the conclusion that ECG is of limited value or even obsolete for LVH detection is not true. Based on the fact that ECG does not estimate mass, we come to the wrong conclusion that ECG is not suitable for LVH diagnostics. What could be more precisely concluded is the statement that ECG is not suitable for the estimation of size of the left ventricle. However, we know that the primary information given by electrocardiography is not the information about size, but about electrical properties of the myocardium. So, we are back at the point of asking unanswerable question, and we are going around in circles.

\section{Alternative conclusions}

Based on the recognition of the unstated assumptions, their re-evaluation, and on the effort to avoid possible flaws, we have developed alternative conclusions:

Because the increased amplitude of the QRS complex is recorded only in a smaller proportion of patients with anatomical LVH, the dominant proportion of patients with anatomical LVH deviates from this expected increase in QRS amplitude. In other words, their QRS amplitude is lower than that expected. These findings are not "false", they are "true", because they are the results of an objective diagnostic method, and in our previous works we called these deviations the "relative voltage deficit" 58,59. Taking into account the difference between the expected voltage related to increased LVM and the actual recorded voltage, it would be appropriate to call this difference a "relative voltage deficit". This term indicates that the recorded QRS voltage in a particular patient with echocardiographically assessed LVH is lower than expected. And, contrary to the term "false-negative", which implicitly neglects the results of ECG evaluations by earmarking them as "false" it imposes the need for further diagnostic clarification.

This relative voltage deficit is conditioned by changes in nonspatial determinants, by changed active and passive electrical properties of the hypertrophied myocardium as compared to healthy tissue.

These changes are not linear during the progression of LVH. They are not proportional to the increase of LVM. In other words, the QRS amplitude does not follow the severity of LVH and the increase of LVM proportionally.

The relative voltage deficit can be enhanced by other associated cardiac pathologies and can be modified by therapy.

The recognition and quantification of the relative voltage deficit as a deviation from normal could have diagnostic and prognostic value and importance.

To quantify the relative voltage deficit, we used a new parameter: a ratio of QRS voltage to left ventricular size ${ }^{60}$. This ratio was called the specific potential of myocardium (SP). Using left ventricular size as a denominator, the voltage is indexed to a unit of myocardium and the influence of left ventricular size on the voltage magnitude is taken into account. In this manner, the relative voltages related to equal units of myocardium can be compared. The SP value can be understood as a potential contribution of one unit of myocardial tissue of the left ventricle to the total cardioelectric field. In terms of solid-angle theory, it can be considered a summary parameter of nonspatial determinants.

In our previous works, we found decreased values of SP in hypertensive patients as compared with those in healthy subjects ${ }^{61}$. Decreased $\mathrm{SP}$ values were observed in experimental $\mathrm{LVH}$ due to volume overload in rabbits ${ }^{62}$. A maxi- 
mum decrease was recorded during the first and the third stages of experimental LVH. The SP values did not reach the control values even in the second stage when the maximum increase in QRS amplitudes was observed. Similarly, decreased SP values were found in spontaneously hypertensive rats in the period of the initial increase in blood pressure when compared with age- and sex-matched healthy WistarKyoto rats ${ }^{63}$.

\section{The decision to apply the conclusions to patients' health care}

ECG is an easily available and affordable method and provides a lot of information on the status of the heart. However, the impact of increased QRS amplitude on clinical decision making with respect to diagnostic classification, selection of therapy and prognosis is surprisingly rather limited.

As already mentioned, only increased QRS amplitude in defined leads is considered a "positive" finding, ie, evidence of LVH, and only these findings are used for clinical decision making. The results within normal limits are considered "negative" and are not taken into account. When confronted with the gold standard, for example with echocardiography, both positive and negative ECG results are, in cases of discrepancies between these 2 methods, considered "false", in other words "wrong".

Electrocardiographically detected LVH has been shown to be a strong independent predictor of cardiac morbidity and mortality both in patients with essential hypertension and in the general population, as well as in normotensive adults ${ }^{24,64,65}$.

In hypertensive patients, the ECG signs of LVH are considered an indicator of target organ damage and are used for further diagnostic decision making. The WHO classification ${ }^{66}$ of the severity of hypertension uses the presence or absence of electrocardiographic LVH as a criterion for differentiating between stages 1 and 2 of established hypertension, even in cases where no signs of LVH are present on the echocardiogram. Similarly, it is included in the risk stratification of hypertensive patients recommended by $\mathrm{JNC} \mathrm{VI}^{67}$. The practical consequence for an individual patient is the recommendation to begin the pharmacotherapy without waiting for the effect of nonpharmacological intervention. However, the finding of an increased QRS voltage has no impact on the selection of therapy.

In spite of the evidence, the importance of the ECG signs in patients with mild to moderate essential hypertension is marginalized because of the low incidence and low sensitivity of ECG findings ${ }^{34,35,68,69}$. In the general population, ECG signs of LVH are rarely seen in calculations of risk scores in health promotion programs.

The so-called false-negative results, ie, those with increased LVM andECG amplitude within normal limits, are not considered for further diagnostic or prognostic decision making and have no impact on the choice of therapy. However, these false-negative findings prevail. Hearts with hypertrophied left ventricles but without the expected pro- portional increase in QRS amplitude are frequent findings in clinical practice, and the high number of false-negative results indicates the need for assessing the diagnostic and prognostic significance of this phenomenon.

In summary, the following gaps in knowledge can be identified with respect to the application of the increased QRS amplitude for diagnostic decision making as regards the individual patient: what are the diagnostic meaning and the consequence of further diagnostic procedures in cases of an isolated increase in QRS amplitude in an eventually healthy population, or in combination with cardiac pathologies other than hypertension? What is the consequence of an isolated increase in QRS amplitude for the selection of therapy and monitoring its effect on hypertension and other cardiac pathologies? What are the diagnostic meaning and the consequences of further diagnostic procedures for finding the discrepancy-increased LVM and low voltage of the relative voltage deficit? What is the consequence of finding the relative voltage deficit for the selection of therapy and monitoring the effect of therapy? Is the prognostic and diagnostic meaning of the voltage deficit different from the so-called true-positive results, where ECG and echocardiographic findings are in agreement?

\section{Conclusion}

The current ECG diagnostics of LVH based on voltage criteria aim to estimate LVM. Its underlying hypothesis includes unstated assumptions about the nonspatial determinants of QRS voltage: First, the electrical properties of hypertrophied myocardium do not differ from that in healthy ones, and second, they do not change during the development and progression of LVH. Because these 2 conditions are not true, the performance of the voltage criteria is limited.

The re-evaluation of the hypothesis is based on a contingency approach-and considers also findings of relatively lower ('normal') QRS voltage in hypertrophied left ventricles: so-called false-negative results, as a result of the relative voltage deficit due to complex changes in the electrical properties of hypertrophied myocardium. Hypertrophied left ventricles with relative voltage deficits, and changes in the relative voltage deficit over time or with treatment, seem to have diagnostic and prognostic importance.

Finally, the results of the application of EBM to electrocardiography example of the ECG diagnostics of LVH allows us to conclude that the application of the EBM approach has contributed to revealing old habits and inertia in thinking fixed during the hundred years and of gaps in our knowledge: an unanswerable question is asked; the evidence is modified slightly or considerably by re-quotations; the validity of ECG as a diagnostic test is tested in relation to only 1 characteristic of LVH size. It follows that the traditionally used gold standard methods are questionable or even inappropriate. Sensitivity and specificity reflect the association with size but not the primary information provided by electrocardiography; the evidence is biased by selection of patients; the dominant findings, the still so-called false- 
negative results, are neglected in diagnostics; the application of the theoretical background, the spatial angle theory, is only partial; gaps exist in our knowledge with respect to the clinical meaning and importance of findings, when the size and the electrical properties are not in accordance, to the still so-called false-negative and false-positive findings. Furthermore, we do not know whether they differ in their clinical importance from those designated as true-positive; gaps exist in our knowledge with respect to the changes in the relationship between size and electrogenesis during the progression of LVH and with therapy.

The EBM approach has contributed to developing an alternative view of the possibilities of electrocardiography in LVH diagnostics, and this is the main lesson we can learn from EBM. It has been shown that the EBM approach is a powerful tool for re-evaluation of old hypotheses in the context of the contemporary knowledge, a tool for generating new ideas and directions for research. Perhaps, in the case of ECG, and not only for LVH, it may contribute to the identification of the role of ECG and to the added value of ECG in clinical diagnostics.

\section{Acknowledgements}

This study was supported in part by grant $1 / 7189 / 20$ from The Science Grant Agency (VEGA), Slovak Republic.

\section{References}

1. Mirvis DM. What's wrong with electrocardiography? J Electrocardiol 1998; 31: 313-316.

2. Sackett DL, Rosenberg WMC, Gray JAM, Haynes RB, Richardson WS. Evidence based medicine: what is and what it isn't. Br Med J 1996; 312: 71 -2.

3. Warren S. Neoplasm. In: Anderson WAD (ed.). Pathology. St. Louis: The CV Mosby Co., 1953: 423-48.

4. Meerson FZ. The myocardium in hyperfunction, hypertrophy and heart failure. Circ Res 1969; 25(Suppl. 2): 1-163.

5. Fizel A, Fizelova A, Turcany M. The relations between ultrastructural and metabolic changes of the myocardium of the hypertrophied and failing heart (Vztah medzi ultrasturkturalnymi a metabolickymi zmemani myokardu hypertrofovaneho a zlyhavajuceho srdca). Folia Fac Med Univ Comeniane Bratisl 1984; 22: 9-119.

6. Davies MJ. Gross morphology of cardiac hypertrophy. In: Sheridan DJ (ed.). Left Ventricular Hypertrophy. London: Churchill Livingstone, 1998: 17-22.

7. Sokolow M, Lyon TP. The ventricular complex in left ventricular hypertrophy as obtained by unipolar precordial and limb leads. Am Heart J 1949; 37: 161-86.

8. Wolff L. Fundamentals and Clinical Applications. $3^{\text {rd }}$ ed. Philadelphia: WB Saunders, 1962.

9. Romhilt DW, Estes EH Jr. A point score system for the ECG diagnosis of left ventricular hypertrophy. Am Heart J 1968; 75: 752-8.

10. Casale PN, Devereux RB, Kligfield P, et al. Electrocardiographic detection of left ventricular hypertrophy: development and prospective validation of improved criteria. J Am Coll Cardiol 1985; 6: 572-80.

11. Arnsdorf MF. Electrophysiology of the heart. Electrocardiography: Fundamental Theory. Part 1. American Physiological Society, 1978: 1108.

12. Liu JE, Devereux RB. Clinical assessment of cardiac hypertrophy. In: Sheridan DJ, (ed). Left Ventricular Hypertrophy. London: Churchill Livingstone, 1998: 11-6.

13. Bayley RH. Biophysical Principles of Electrocardiography. New York: Paul B. Hoeber, 1958.

14. Scher AM. Trends in electrocardiographic recording. In: Schlant RC, Hurst JW (eds). Advances in Electrocardiography. New York: Grune \& Stratton, 1972: 1-8.

15. ReichekN, Devereux RB. Left ventricular hypertrophy: relationship of anatomic, echocardiographic and electrocardiographic findings. Circulation 1981; 63: 1391-8.

16. Einthoven W. Telecardiogramme. Arch Int Physiol 1906-1907; 4: 132-63.

17. Brody DA. The theoretical analysis of intracavitary blood mass influence on the heart-lead relationship. Circ Res 1956; 4: 731-8.

18. Feldman T, Borow KM, Neumann A, Lang RM, Childers RW. Relation of electrocardiographic R-wave amplitude to changes in left ventricular chamber size and position in normal subjects. Am J Cardiol 1985; 55: 1168-74.

19. Talbot S, Kilpatrick D, Jonathan A, Raphael MJ. QRS voltage of the electrocardiogram and Frank vectorcardiogram in relation to ventricular volume. Br Heart J 1977; 39: 1109-13.

20. Fuenmayor AJ, Vasquez CJ, Fuenmayor AM, Winterdaal DM, Rodriguez D. Hemodialysis changes the QRS amplitude in the electrocardiogram Int J Cardiol 1993; 41: 141-5.

21. Vancheri F, Barberi O. Relationship of QRS amplitude to left ventricular dimensions after acute blood volume reduction in normal subjects. Eur Heart J 1989; 10: $341-5$.

22. Vitolo E, Madoi S, Palvarine M, et al. Relationship between changes in R wave voltage and cardiac volumes. A vectorcardiographic study during hemodialy sis. J Electrocardiol 1987; 20: 138-46.

23. Antman EM, Green LH, Grossman W. Physiologic determinants of the electrocardiographic diagnosis of left ventricular hypertrophy. Circulation 1979; 69: 386-96.

24. Kannel WB, Gordon T, Castelli WP, Margolis JR. Electrocardiographic left ventricular hypertrophy and risk of coronary heart disease: The Framingham Study. Ann Intern Med 1970; 72: 813-22.

25. Okin PM, Roman MJ, Devereux RB, Kligfield P. Electrocardiographic identification of increased left ventricular mass by simple voltage-duration products. J Am Coll Cardiol 1995; 25: 417-23.

26. Romhilt DW, Bove KE, Norris RJ, et al. A critical appraisal of the electrocardiographic criteria for the diagnosis of left ventricular hypertrophy. Circulation 1969; 40: 185-95

27. Kimura M, Matsushita S, Nakahara K, Miyakawa A, Kuramoto K. Evaluation of electrocardiographic criteria for left ventricular hypertrophy based on anatomical comparison. J Electrocardiol 1987; 20: 369-74.

28. Timmis GC, Bakalyar DM, Gordon S. Accuracy of computerized electrocardiographic identification of left ventricular hypertrophy as determined by echocardiographic measurements of left ventricular mass: Evaluation of a widely used computer program. J Am Coll Cardiol 1986; 8: 301-9.

29. Mashima S. Theoretical considerations on the electrocardiogram of ventricular hypertrophy. J Electrocardiol 1976; 9: 133-8.

30. Roberts DE, Hersh LT, Scher AM. Influence of cardiac fiber orientation on wavefront voltage, conduction velocity, and tissue resistivity in the dog. Circ Res 1979; 44: 701- 12 .

31. Saffitz JE, Schuessler RB, Yamada KA. Mechanisms of remodelling of gap junction distributions and the development of anatomic substrates of arrhythmias. Cardiovasc Res 1999; 42: 309-17.

32. Natte S, Roden DM, Escande D. A spotlight on electrophysiological remodelling and the molecular biology of ion channels. Cardiovasc Res 1999; 42: 267-9.

33. Fisch $\mathrm{CH}$. Electrocardiography and vectorcardiography. In: Braunwald E (ed). Heart Disease. $4^{\text {th }}$ ed. Philadelphia: WB Saunders, 1992: 116-61.

34. Savage DD, Drayer JIM, Henry WL, et al. Echocardiographic assessment of cardiac anatomy and function in hypertensive subjects. Circulation 1979; 59: 623-32.

35. Devereux RB, Casale PN, Wallerson DC, et al. Cost-effectiveness of echocardiography and electrocardiography for detection of left ventricular hypertrophy in patients with systemic hypertension. Hypertension 1987; 9(2):II69-76.

36. Devereux RB, Reichek N. Echocardiographic determination of left ventricular mass in man: anatomic validation of the method. Circulation 1977; 55: 613-8.

37. Devereux RB, Alonso DR, Lutas EM, et al. Echocardiographic assessment of left ventricular hypertrophy: comparison to necropsy findings. Am J Cardiol 1986; 57: 450-8.

38. Molloy TJ, Okin PM, Deverex RB, Kligfield P. Electrocardiographic detection of left ventricular hypertrophy by the simple QRS voltage-duration product. J Am Coll Cardiol 1992; 20: 1180-6.

39. Okin PM, Roman MJ, Devereux RB, Kligfield P. Electrocardiographic identification of increased left ventricular mass by simple voltage-duration products. J Am Coll Cardiol 1995; 25: 417-23.

40. Simonson E. Differentiation Between Normal and Abnormal in Electrocardiology. St. Louis: CV Mosby, 1961. 
41. Pipberger HV, Goldman MJ, Littmann, et al. Correlation of the orthogonal electrocardiogram and vectorcardiogram with constitutional variables in 518 normal men. Circulation 1967; 35: 536-51.

42. Ishikawa K. Correlation coefficients for electrocardiographic and constitutional variables. Am Heart J 1976; 92: 152-61.

43. Rautaharju PM, LaCroix AZ, Savage DD, et al. Electrocardiographic estimate of left ventricular mass versus radiographic cardiac size and the risk of cardiovascular disease mortality in the epidemiological follow-up study of the first National Health and Nutrition Examination Survey. Am J Cardiol 1988; 62: 59-66.

44. Odom II H, Davis JL, Dinh HA, et al. QRS voltage measurements in autopsied men free of cardiopulmonary disease: A basis for evaluating total $Q R S$ voltage as an index of left ventricular hypertrophy. Am J Cardiol 1986; 58: 801-4.

45. Murphy ML, Thenabadu N, Blue LR, et al. Descriptive characteristics of the electrocardiogram from autopsied men free of cardiopulmonary disease - a basis for evaluating criteria for ventricular hypertrophy. Am J Cardiol 1983; 52: 1275-80.

46. Uhley HN. Study of the transmembrane action potential, electrogram, electrocardiogram and vectorcardiogram of rats with left ventricular hypertrophy. Am J Cardiol 1961; 7: 211-7.

47. Kaufmann RL, Homburger H, Wirth H. Disorder in excitation-contraction coupling of cardiac muscle from cats with experimentally produced right ventricular hypertrophy. Circ Res 1971; 28: 346-57.

48. Basset AL, Gelband H. Chronic partial occlusion of the pulmonary artery in cats. Change in ventricular action potential configuration during early hypertrophy Circ Res 1973; 32: 15-26.

49. Fry CH, Carey P, Mundy AR, Sheridan DJ. Altered conduction in left ventricular hypertrophy. In: Sheridan J. (ed.). Left Ventricular Hypertrophy. London: Churchill Livingstone, 1998: 113-20.

50. Toyoshima H, Park Y-D, Ishikawa, et al. Effect of ventricular hypertrophy on conduction velocity of activation front in the ventricular myocardium. Am J Cardiol 1982; 49: 1938-45.

51. Winterton SJ, Turner MA, O'Gorman DJ, Flores NA, Sheridan DJ. Hypertrophy causes delayed conduction in human and guinea pig myocardium: accentuation during ischaemic perfusion. Cardiovasc Res 1994; 23: 47-54.

52. Cooklin M, Wallis WRJ, Sheridan DJ, Fry CH. Changes in cell-to-cell electrical coupling associated with left ventricular hypertrophy. Circ Res 1997; 80: 765-71.

53. McIntyre $\mathrm{H}$, Fry $\mathrm{CH}$. Abnormal action potential propagation in isolated human hypertrophied left ventricular myocardium. J Cardiovasc Electrophysiol 1997; 8: 887-94.

54. Carey PA, Cooklin M, Sheridan DJ, Fry CH. Estimation of gap-junction resistance in hypertrophied and hypoxic guinea pig myocardium. J Physiol 1997; 504: 96-7.

55. Peters NS, Green CR, Poole-Wilson PA, Severs NJ. Reduced content of connexin43 gap junctions in ventricular myocardium from hypertrophied and ischaemic human hearts. Circulation 1993; 88: 864-75.

56. Goldfine SM, Walcott B, Brink PR, Magid NM, Borer JS. Myocardial conne- xin43 expression in left ventricular hypertrophy resulting from aortic regurgitation. Cardiovasc Pathol 1999; 8: 1-6.

57. Yamamoto S, James TN, Sawada K-I, Okabe M, Kawamura K. Generation of new intercellular junctions between cardiocytes. A possible mechanism compensating for mechanical overload in the hypertrophied human adult myocardium. Circ Res 1996; 78: 362-70.

58. Bacharova L. Effect of left ventricularhypertrophy on the cardiac electrical field: The concept of the specific potential of myocardium. Exp Clin Cardiol 1998; 3: 128-33.

59. Bacharova L, Kyselovic J. Electrocardiographic diagnosis of left ventricular hypertrophy: Is the method obsolete or should the hypothesis be reconsidered? Med Hypotheses 57; 2001: 487-90.

60. Bacharova L. Reasoning for introducing a new parameter for assessment of myocardial status - the specific potential of myocardium. In: Cohen ME, Hudson DL, (eds.). Comparative Approaches to Medical Reasoning. Singapore: World Scientific Publ Co., 1995: 217-41.

61. Bacharova L, Melotova J, Sedlakova K. The 'specific potential' as a parameter of myocardial changes in left ventricular hypertrophy. In: Abel H. (ed.). Electrocardiology '88. Amsterdam: Excerpta Medica; 1989: 195-8.

62. Bacharova L, Bernadic M, Fizelova A. Electrocardiographic manifestation of experimental left ventricular hypertrophy. In: Jagielski J, Gornicki M. (eds.). Electrocardiology 91. Singapore: World Scientific, 1992: 29-32.

63. Bacharova L, Kyselovic J, Klimas J, Radman A. The decrease of specific potential of myocardium in early stage of experimental hypertension. In: Roshchevsky MP (ed.). Electrocardiology '99. Syktyvkar: Institute of Physiology of the Komi Science Centre, 2000: 30-7.

64. Rautaharju PM, LaCroix A, Savage D, et al. Electrocardiographic estimate of left ventricular mass versus radiographic cardiac size and the risk of cardiovascular disease mortality in the epidemiologic follow-up study of the First National Health and Nutrition Examination Study. Am J Cardiol 1988; 62: 59-66.

65. Brown DW, Giles WH, Croft JB. Left ventricular hypertrophy as a predictor of coronary heart disease mortality and the effect of hypertension. Am Heart J 2000; 14: 848-56.

66. World Health Organization Expert Committee: Arterial hypertension. Geneva: World Health Organization; 1978; 8-10.

67. The Sixth Report of the Joint National Committee on Prevention, Detection, Evaluation, and Treatment of High Blood Pressure. Arch Intern Med 1997; 157: 2413-52.

68. Levy D, Labib SB, Anderson KM, Christiansen JC, Kannel WB, Castelli WP. Determinants of sensitivity and specificity of electrocardiographic criteria for left ventricular hypertrophy. Circulation 1990; 81: 815-20.

69. Hypertension Detection and Follow-up Program Cooperative Group. Five year findings of the Hypertension Detection and Follow-up Program: reduction in mortality of persons with high blood pressure, including mild hypertension. JAMA 1979; 242: 2562-71. 